\title{
let-7b/g silencing activates AKT signaling to promote gastric carcinogenesis
}

\author{
Wei Kang 1,2,3,4, Joanna HM Tong ${ }^{1,2,3}$, Raymond WM Lung 1,2,3, Yujuan Dong ${ }^{2}$, Weiqin Yang ${ }^{5,6}$, Yi Pan 1,2,3, \\ Kin Mang Lau ${ }^{1,3}$, Jun Yu ${ }^{2,5}$, Alfred SL Cheng ${ }^{2,6}$ and Ka Fai To $0^{1,2,3,4^{*}}$
}

\begin{abstract}
Background: Aberrant AKT activation contributes to gastric cancer cell survival and chemotherapy resistance, however its regulation is poorly understood. microRNAs have been established to be important regulators in gastric carcinogenesis. Here, we showed the functional role and putative target of let-7b and let-7g (let-7b/g) in gastric carcinogenesis.

Methods: The expression of let-7b/g in gastric cancer cell lines and primary tumors were evaluated by miRNA qRT-PCR. The putative target gene of let-7b/g was explored by TargetScan followed by further validation. Functional analyses including MTT proliferation, monolayer colony formation, cell invasion assays and in vivo study were performed in both ectopic expression and knockdown approaches.

Results: let-7b/g was found down-regulated in gastric cancer and its downregulation was associated with poor survival and correlated with lymph node metastasis. let-7b/g inhibited AKT2 expression by directly binding to its 3'UTR, reduced p-AKT (S473) activation and suppressed expression of the downstream effector pS6. AKT2 mRNA expression showed negative correlation with the expression of let-7b/g in primary tumors. Short interfering RNA (siRNA) mediated knockdown of AKT2 phenocopied the tumor-suppressive effects of let-7b/g. Moreover, AKT2 re-expression partly abrogated the growth-inhibitory effect of let- $7 \mathrm{~b} / \mathrm{g}$.
\end{abstract}

Conclusion: In conclusion, our findings reveal decreased let-7b/g contributes to aberrant AKT activation in gastric tumorigenesis and provide a potential therapeutic strategy for gastric cancer.

Keywords: let-7b, let-7g, AKT2, Gastric cancer, Tumor suppressor

\section{Background}

Gastric cancer is the fourth most common cancer worldwide, with an estimated one million new cases per year [1]. H. pylori is the most important risk factor in gastric tumorigenesis which induces gastric cancer potentially by chronic inflammation or through the action of H. pylori virulence factors such as CagA [2]. Approximately 95\% of gastric cancer are adenocarcinomas by histological phenotype as intestinal type, diffuse type and mixed/ unclassifiable according to Lauren's classification [3]. Nowadays, several molecular classifications of gastric

\footnotetext{
* Correspondence: kfto@cuhk.edu.hk

'Department of Anatomical and Cellular Pathology, State Key Laboratory in Oncology in South China, Prince of Wales Hospital, The Chinese University of Hong Kong, Hong Kong, SAR, People's Republic of China

${ }^{2}$ Institute of Digestive Disease, Partner State Key Laboratory of Digestive Disease, The Chinese University of Hong Kong, Hong Kong, SAR, People's Republic of China

Full list of author information is available at the end of the article
}

cancer have been proposed based on the analysis of whole-genome gene expression studies or deep sequencing studies [4]. Most gastric cancer patients are diagnosed at the advanced stage often accompanied with extensive invasion and lymphatic metastasis. Thus the investigations into the molecular mechanisms involving in gastric cancer progression become imperative and urgent for targeted therapy.

microRNAs (miRNAs) are a class of small non-proteincoding RNAs which have been identified as a new kind of gene expression regulators through binding to the 3 ' untranslated regions ( 3 'UTRs) of target mRNA, thus blocking mRNA translation or resulting in mRNA degradation $[5,6]$. Emerging evidence shows that miRNAs are abnormally expressed in cancer development and the deregulated miRNAs are associated with tumor initiation, promotion and progression by regulating target genes expression [7]. Some miRNAs such as 
miR-372, -544, -25, -373 show up-regulated expression and exert oncogenic property in gastric cancer [8-10]. On the contrary, miR-9, -202-3p, -7 and -206 are downregulated and play tumor suppressor function in gastric carcinogenesis [11-14].

By using miRNA expression microarray, we have identified aberrantly expressed miRNAs in gastric cancer cell lines including let-7 family. Compared with normal gastric epithelium tissue, let-7 family was found to be downregulated in all 9 gastric cancer cell lines (Additional file 1: Table S1), in which let-7a/b/f and let-7c/d/e/g/i were down-regulated for thousand and hundred times respectively. let-7 microRNA was first identified in gastric cancer due to its targeting on high mobility group A2 (HMGA2) [15], a nonhistone chromosomal protein that can modulate transcription by altering chromatin architecture. It has been reported that the expression of let- 7 in gastric cancer correlated with $H$. pylori infection [16]. Hayashi reported that $H$. pylori CagA induced aberrant epigenetic silencing of let-7 expression [17]. let-7f, a member of let-7 family, is able to inhibit tumor invasion and metastasis by targeting MYH9 in human gastric cancer [18]. However, for let-7b and let-7g (let-7b/g), the possible role and putative target genes in gastric cancer cells are still not well elucidated and need investigation.

\section{Materials and methods}

\section{Cell line and primary gastric tissues}

The information of human gastric cancer cell lines (MKN1, MKN7, MKN28, MKN45, SNU1, SNU16, AGS, KatoIII, NCI-N87) were previously described [19]. Cells were maintained in RPMI 1640 medium (GIBCO, Grand Island, NY) supplemented with $10 \%$ fetal bovine serum (GIBCO, Grand Island, NY). Among them, AGS (poorly differentiated and p53-wide type), NCI-N87 (well differentiated and p53-mutation) and MKN45 (with xenograft formation ability) were representatively chosen for functional studies.

The primary paired samples from gastric cancer patients were randomly selected from Prince of Wales Hospital (Year 1999-2010). All participants provided written consent for the research experiment. Ethical approval was obtained from the Joint Chinese University of Hong Kong-New Territories East Cluster Clinical Research Ethics Committee (CREC Ref. No.2009.521).

\section{RNA extraction and quantitative real-time polymerase chain reaction ( $q R T-P C R$ )}

The normal tissue corresponds to Human Stomach Total RNA commercially available from Ambion (AM7996, Grand Island, NY). A total of 67 paired (tumor and corresponding adjacent non-tumorous tissue) RNA samples were achieved from frozen tissues and 9 paired samples were got from micro-dissection of paraffin embedded tissues. RNA was extracted using TRIzol reagent (Invitrogen, Grand Island, NY). High Capacity cDNA Reverse Transcription Kits (Applied Biosystems, Grand Island, NY) were used for cDNA synthesis. qRT-PCR was used to quantitative differences in mRNA expression of AKT2 and primers were as following (sense-Exon 9: CAA AGA TGG CCA CAT CAA GA; anti-sense-Exon 10: GTC ATT GTC CTC CAG CAC CT). AKT1 expression were detected using the following primers (sense-Exon 9: GAG ATT GTG TCA GCC CTG GA; anti-sense-Exon 10: AGC CCG AAG TCT GTG ATC TT). The relative expression level was normalized by RPL29 (sense-Exon 3: GGA CCC CAA GTT CCT GAG G; anti-sense-Exon 4: GCA TTG TTG GCC TGC ATC TT) in gastric tissues and B2M (senseExon 1: ACT CTC TCT TTC TGG CCT GG; antisense-Exon 2: ATG TCG GAT GGA TGA AAC CC) in gastric cancer cell lines [20]. PCR was performed using SYBR Green PCR reagents (Applied Biosystems) according to the manufacturer's instructions. The reactions were incubated in a 96 -well plate at $95^{\circ} \mathrm{C}$ for $10 \mathrm{~min}$, followed by 40 cycles of $95^{\circ} \mathrm{C}$ for 15 seconds and $60^{\circ} \mathrm{C}$ for 1 minute.

For miRNA expression detection, Taqman miRNA assays were used to quantify the expression levels of mature let-7b and let-7g (KIT, 002619 and 002282, Applied Biosystems). The relative expression level of microRNAs was normalized by RNU6B (KIT, 001093, Applied Biosystems). The reactions were performed in 7500 Fast Real-Time System (Applied Biosystems) and the reaction mix was incubated at $95^{\circ} \mathrm{C}$ for 30 seconds, followed by 40 cycles of $95^{\circ} \mathrm{C}$ for 8 seconds and $60^{\circ} \mathrm{C}$ for 30 seconds.

\section{Protein extraction and Western blot analysis}

Protein was extracted from gastric cancer cell lines and paired primary tissues using RIPA lysis buffer with proteinase inhibitor. Protein concentration was measured by the method of Bradford (Bio-Rad, Hercules, CA) and $20 \mu \mathrm{g}$ of protein mixed with $2 \times$ SDS loading buffer was loaded per lane, separated by $12 \%$ SDS-polyacrylamide gel electrophoresis. Protein expression was detected using primary monoclonal anti-AKT2 antibody (1:1000 dilution, \#3063, Cell Signaling, Danvers, MA), anti-phospho-AKT (S473) (1:1000 dilution, \#9271, Cell Signaling) and anti-pS6 antibody (1:1000, \#4858, Cell Signaling). The secondary antibodies were anti-Mouse IgG-HRP (1:30000, 00049039, Dako, Glostrup, Denmark) and anti-Rabbit IgG-HRP (1:10000, 00028856, Dako). The Western blot bands were quantified by ImageJ.

\section{miRNA/siRNA (small inference RNA) transfection and functional study}

The miRNA precursors, let-7b (PM11050), let-7g (PM11758), scramble control (AM17110) were purchased from Applied Biosystems. And siAKT2 (SI00287672 and SI00287679) were obtained from Qiagen (Valencia, CA). 
All transfection were performed using Lipofectamine 2000 Transfection Reagent (Invitrogen) in a $20 \mathrm{nM}$ concentration for 48 hours followed with functional study and RNA/ protein analysis. Cell proliferation was assessed using CellTiter 96 Non-Radioactive Cell Proliferation Assay (Promega, Madison, WI) according to manufacturer's instruction. For colony formation assays in monolayer cultures, the transfected cells were cultured in 6-well plates for 10 days. Cells were fixed with $70 \%$ ethanol for 15 minutes and stained with $2 \%$ crystal violet. The cell invasion assays using BD Biocoat Matrigel Invasion Chambers (BD Biosciences, Franklin Lakes, NJ) has been described previously by W. Kang [21]. The experiment was performed in triplicate and the mean including SDs was plotted.

\section{Rescue experiments}

let-7b and let-7g precursors together with the negative control were transfected in AGS, NCI-N87 and MKN45 cells. And 24 hours after precursor transfection, AKT2 expression plasmid (from Addgene, Plasmid \#16000) and empty plasmid (pcDNA3, Life Technologies, Grand Island, NY) were subsequently transfected with FuGENE HD Transfection Reagent (Roche, Nutley, NJ). After another 24 hours, cells were collected for functional study (MTT proliferation assays, monolayer colony formation assays and in vivo animal model). The experiments were repeated for 3 times and the representative data was shown.

\section{Luciferase assays}

The annealed oligonucleotides containing the putative let-7b/g binding site was cloned into pMIR-REPORT vector (Ambion) via HindIII and SpeI. The sequences of oligonucleotides were (sense) AGA CAC TAG TTA GCA CTT CAC ACC CAT TGA and (antisense) GAG AAA GCT TCT AGA GAT TAC AGG CAT GAG CCA CT). Mutations located on the predicted seed binding region were introduced into the luciferase reporter vector using

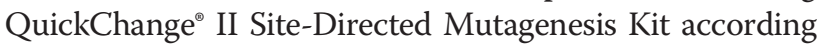
to the manufacturer's instruction. The primer sequences for mutagenesis were 5'-GTG TGG GCA CAG GCC TGG GTT CGT GAT CTT TTT AGT GCC TCT C-3' and 5'-GAG AGG CAC TAA AAA GAT CAC GAA CCC AGG CCT GTG CCC ACA C-3'. The firefly luciferase reporter construct and the control Renilla luciferase construct were co-transfected into NCI-N87 cells in 24-well plates. Cells were harvested after 36 hours for reporter activity analysis using Dual Luciferase Reporter assays (Promega, Madison, WI) as described previously [22].

\section{In vivo tumorigenicity study}

Total three batches of animal models were used in this study. For let-7b/g or siAKT2 functional study, MKN45 cells were transfected with let-7b/g precursors or siAKT2 together with their control counterparts. The cells $\left(5 \times 10^{6}\right.$ cells suspended in $0.1 \mathrm{ml}$ PBS) were injected subcutaneously into the dorsal flank of eight 4-week-old male Balb/c nude mice. For the rescue in vivo study, MKN45 was transfected with let-7b/g for 24 hours following by AKT2 or empty vector transfection for another 24 hours. Then the cells were injected subcutaneously into the dorsal flank of eight Balb/c nude mice. Tumor diameter was measured and documented every 4 days until the tumor reached $10 \mathrm{~mm}$ in diameter. Tumor volume $\left(\mathrm{mm}^{3}\right)$ was estimated by measuring the longest and shortest diameter of the tumor and calculated using the following formula: volume $=(\text { shortest diameter })^{2} \times($ longest diameter $) \times 0.5$ [23]. All animal handling and experimental procedures were approved by Department of Health, Hong Kong (Reference No: 12-241 in DH/HA\&P/8/2/1).

\section{Statistical analysis}

The Student $T$ test was employed to compare the functional effect between the target transfectants and the controls. Expression of let-7b/g and AKT2 (mRNA and protein) in primary cancerous tissues and their corresponding paired noncancerous tissues were compared by paired $T$ test. Correlations between let-7b/g expression and clinicopathologic parameters were assessed by Pearson correlation analysis. The let-7b/g expression in gastric cancer cell lines was compared with it copy number change by non-parametric Spearman's rho rank test. The KaplanMeier method was employed to estimate the survival rates for each variable. The equivalences of the survival curves were tested by log-rank statistics. All statistical analysis was performed by SPSS software (version 16.0; SPSS Inc). A two-tailed $P$ value of less than 0.05 was considered statistically significant.

\section{Results}

\section{The expression of let-7b/g is down-regulated in gastric} cancer and correlates with poor survival

The expression of let-7b/g was measured in gastric cancer cell lines and normal stomach epithelium tissue by miRNA qRT-PCR. let-7b and let-7g were observed downregulated in 6 and 7 gastric cancer cell lines respectively compared with normal gastric epithelial sample (Figure 1A). In a total of 76 paired primary RNA samples, the expression of let-7b and let-7g showed a decreased level in tumors compared with the corresponding nontumorous gastric mucosal samples (let-7b, $P=0.013$; let-7g, $P=0.003$; Figure $1 \mathrm{~B}$ ).

In 76 paired primary samples, let-7b was found downregulated in $53(69.7 \%)$ tumor tissues which normalized by its expression in adjacent normal gastric tissue. Then two groups were stratified according to the median expression of let-7b and each group had the same 38 cases. The low-expression of let-7b group showed a poor survival 


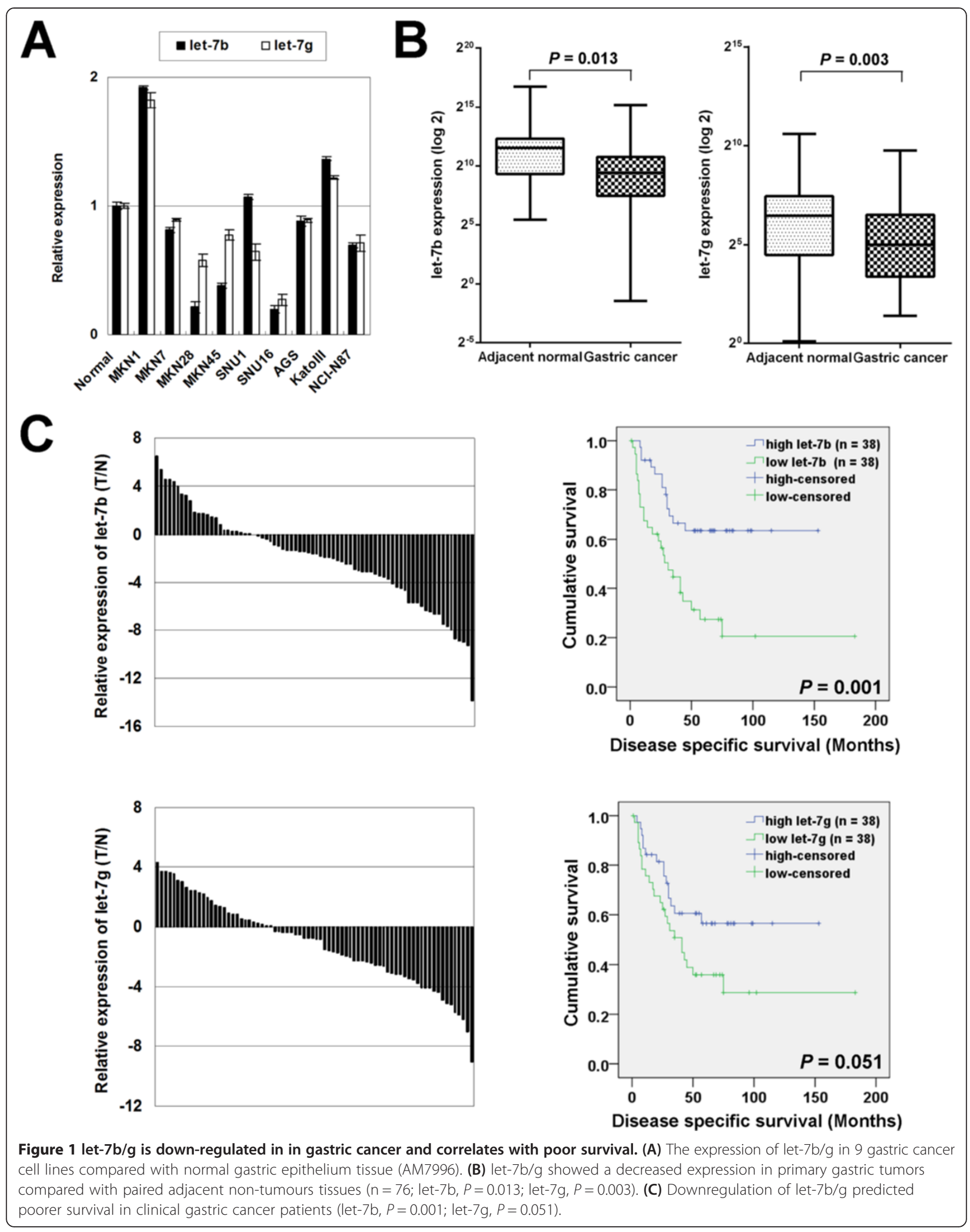


compared with high-expression group $(P=0.001$, up panel of Figure $1 \mathrm{C})$. Meanwhile let-7g showed decreased expression in 48 (63.2\%) tumor tissues when compared with adjacent normal tissues and its downregulation also predicted poorer prognosis in gastric cancer with a marginal significant $P$-value $(P=0.051$, lower panel of Figure $1 C)$. We further investigated the clinicopathologic correlation of let-7b and let-7g. Additional file 2: Table S2 summarized the correlation of let-7b/g with other clinicopathologic parameters in gastric cancer patients. The decreased expression of let$7 \mathrm{~b}$ was correlated with lymph node metastasis $(P=0.021)$. The let-7g downregulation was also associated with N-stage $(P=0.044)$ and lymph node metastasis $(P=0.009)$, suggesting that these two miRNAs might be involved in gastric cancer cell metastasis.

\section{let-7b and let-7g exert tumor suppressor function in} gastric cancer cells

To investigate the functional role of let-7b/g, the precursors of these two miRNAs were transfected into AGS, NCI-N87 and MKN45 cells. let-7b/g suppressed the growth of gastric cancer cells AGS, NCI-N87 and MKN45 as demonstrated by a 5 -day MTT assay $(P<0.001$, Figure $2 \mathrm{~A})$. The tumor suppressor function of let- $7 \mathrm{~b} / \mathrm{g}$ was further validated by monolayer colony formation. A significant reduction of colony number was observed in let-7b/g transfectants
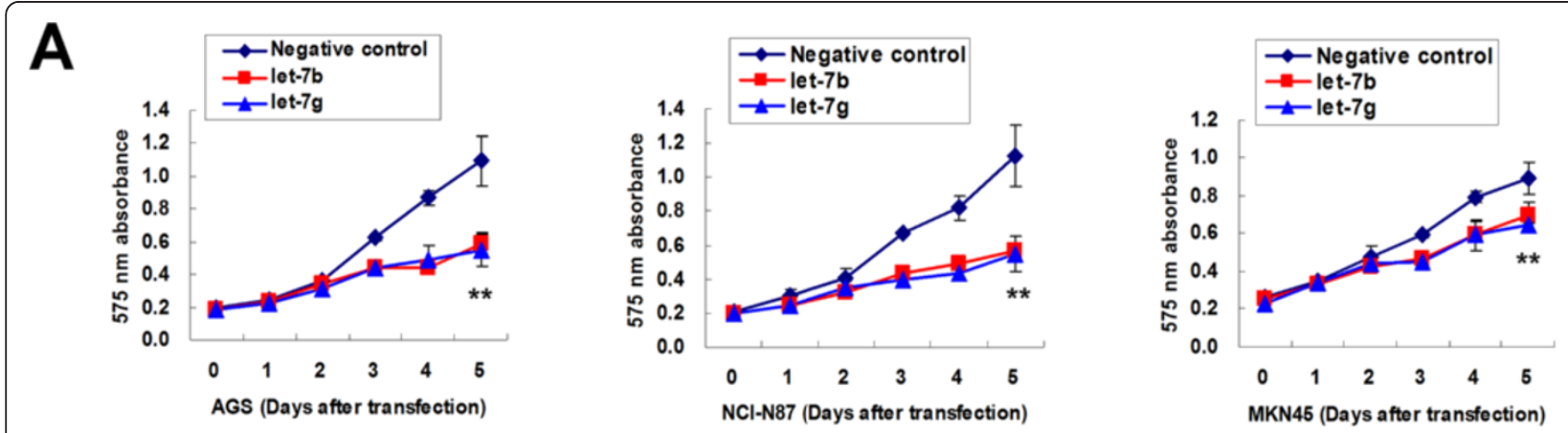

B
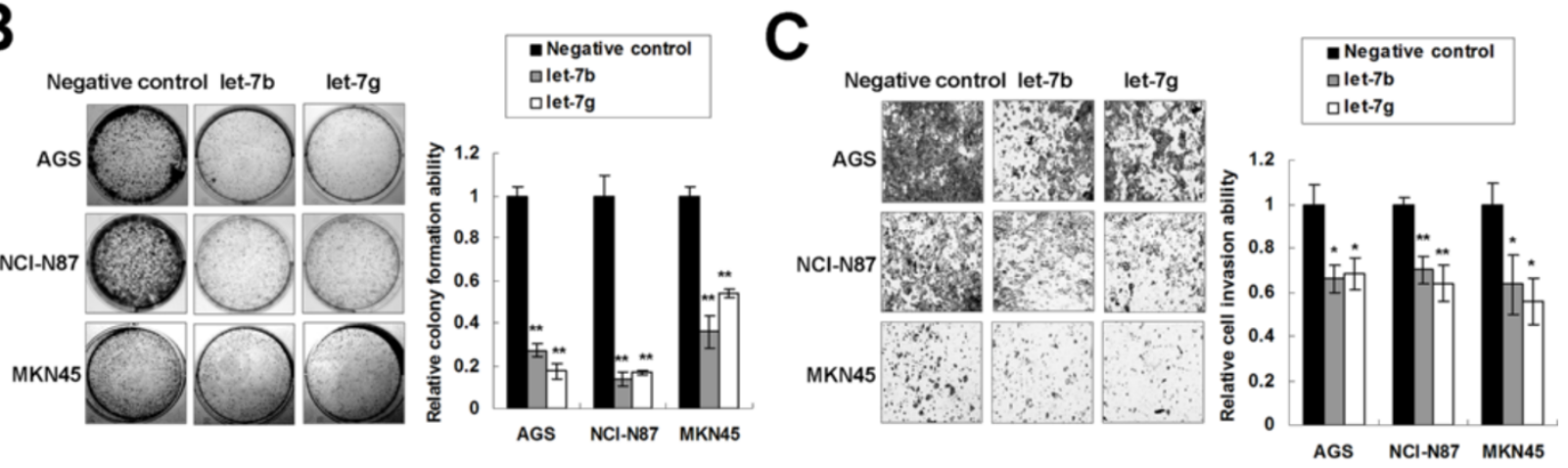

D
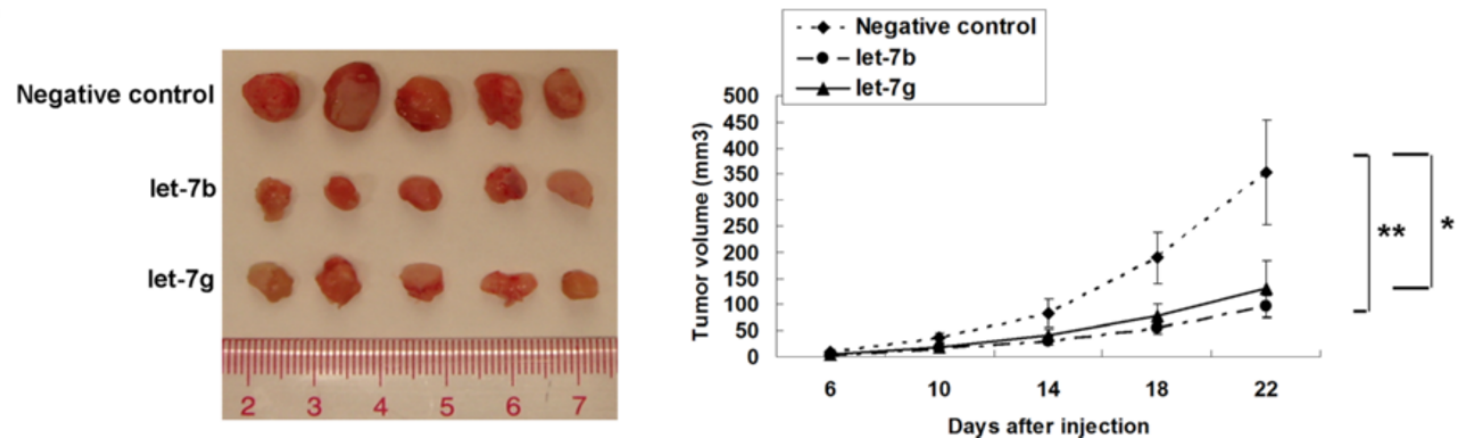

Figure 2 Ectopic expression of let-7b/g exerts tumor suppresser function in gastric cancer cells. (A) 5-day MTT proliferation results of let-7b/g in AGS, NCI-N87 and MKN45 cells (**, P<0.001). (B) let-7b/g decreased monolayer colony formation in AGS, NCI-N87 and MKN45 cells $(* *, P<0.001)$. The experiment was performed in triplicate wells to get standard deviations (SDs). (C) let-7b/g inhibited gastric cancer cell invasion $\left({ }^{*}, P<0.05 ; * *, P<0.001\right)$. Three random vision fields were selected for invaded cell counting to get SDs. (D) let-7b/g-MKN45 formed smaller xenografts than the negative control group $\left({ }^{*}, P<0.05 ;{ }^{*}, P<0.001\right)$. 
compared with scramble miRNA groups $(P<0.001$, Figure $2 \mathrm{~B})$. Ectopic expression of let-7b or let- $7 \mathrm{~g}$ was further revealed to inhibit the invasive capacity of gastric cancer cells as demonstrated by Matrigel invasion assays $(P<0.05$, Figure $2 C)$. The MKN45 cells with let$7 \mathrm{~b} / \mathrm{g}$ transfection were injected subcutaneously into the dorsal flank of nude mice. let-7b/g transfectants formed smaller xenografts than those scramble transfectants after 22 days (let-7b, $P<0.001$; let-7g, $P<0.05$; Figure 2D).

let-7b and let-7g decrease AKT2 expression by direct binding with its $3^{\prime} \mathrm{UTR}$

AKT2 was predicted to be a putative target of let- $7 \mathrm{~b} / \mathrm{g}$ by TargetScan (www.targetscan.org). The putative binding sites of let-7b/g within the 3'UTR of AKT2 were shown in Figure 3A. We transfected let-7b/g into gastric cancer cells and decreased AKT2 mRNA expression level was observed in AGS, NCI-N87 and MKN45 cells $(P<0.05$; left bar chart of Figure 3B). However, ectopic expression of let-7b/g did not change the AKT1 mRNA expression (right bar chart of Figure 3B), indicating let-7b/g only exerts its inhibitory effect on AKT2 but not on AKT1. AKT2 protein showed a decrease expression after ectopic let-7b/g expression (Figure 3C), suggesting that let-7b/g triggered a silencing effect on the endogenous AKT2 expression. Meanwhile, the phosphorylated AKT (S473) and its direct downstream effector of AKT-mTOR pathway, pS6, showed decreased expression upon let-7b/g overexpression. A luciferase reporter assay was performed to

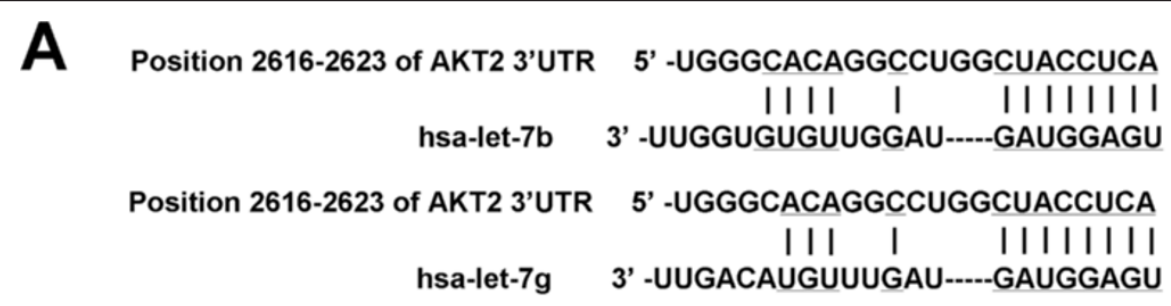

B
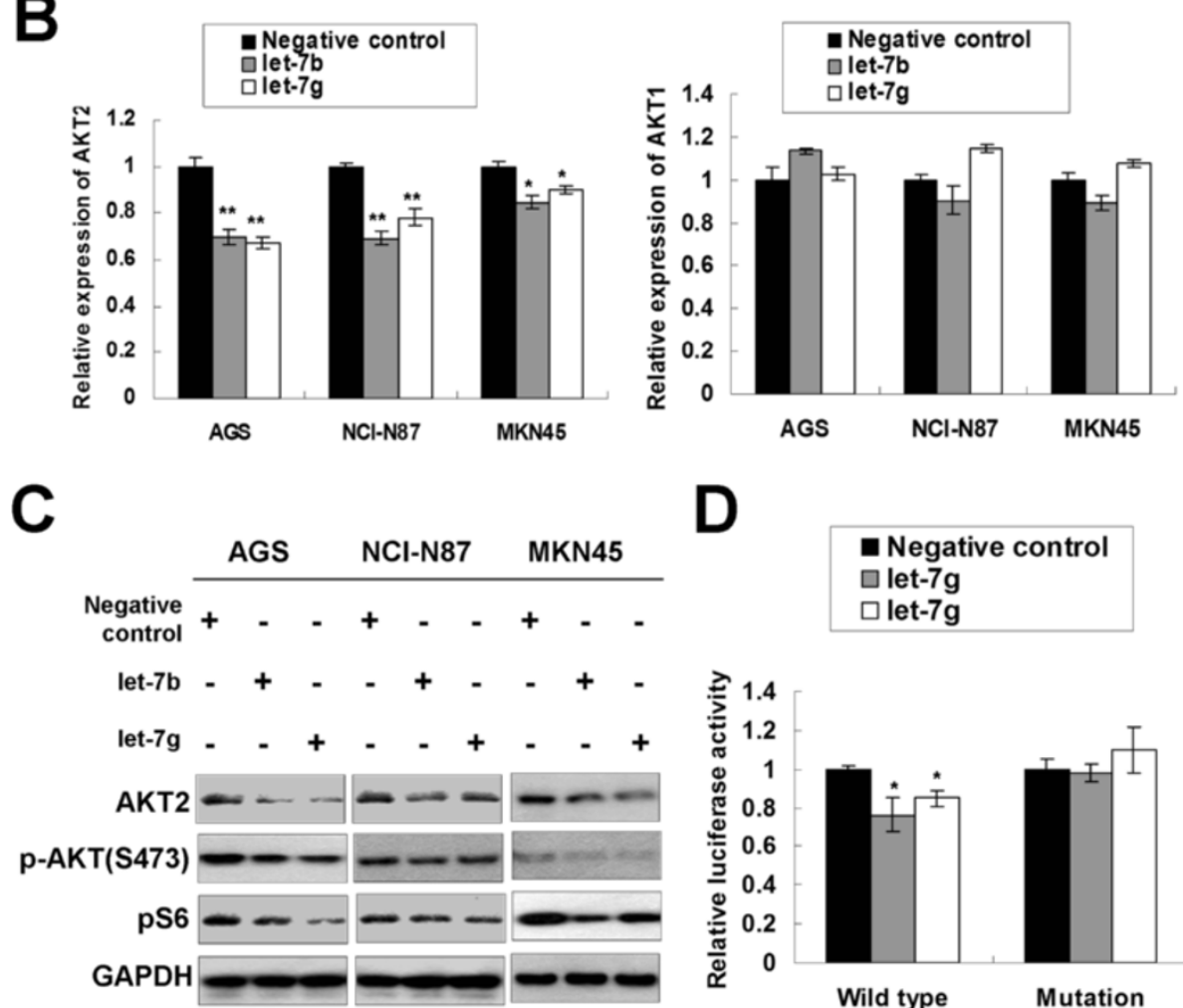

D

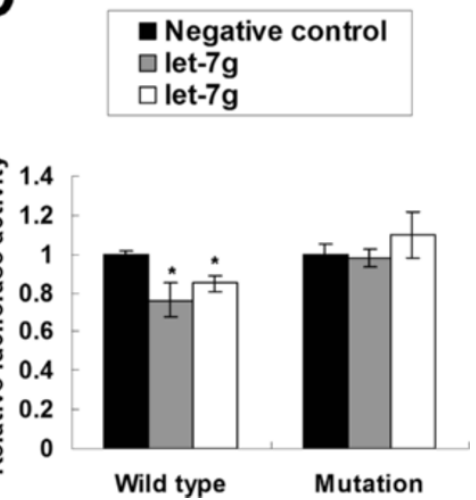

Figure 3 let-7b/g targets AKT2 in gastric cancer. (A) The binding sites of let-7b/g on the AKT2 3'UTR as predicted by TargetScan (www.targetscan.org). (B) AKT2 mRNA expression was down-regulated upon ectopic let-7b/g expression ( ${ }^{*}, P<0.05 ;{ }^{* *}, P<0.001$ ). (C) Western blot analysis of AKT2, $P$-AKT (S473) and pS6 upon let-7b/g ectopic expression in AGS, NCI-N87 and MKN45 cells. (D) Relative luciferase activity in the constructs containing wild-type binding site or mutated binding site of AKT2 $3^{\prime} U T R$ after let-7b/g transfection (Wild type, the construct containing the complementary sequence of seed region; Mutation, the binding site was mutated; $\left.{ }^{*}, P<0.05\right)$. 
test whether AKT2 is a direct target of let-7b/g. As shown in Figure 3D, let-7b/g decreased the relative luciferase activity of constructs encompassing AKT2 3' UTR binding sites in NCI-N87 cells $(P<0.05)$. Meanwhile, let-7b/g did not suppress the luciferase activity in the constructs containing the mutated binding site. The results in this part revealed that let- $7 \mathrm{~b} / \mathrm{g}$ specifically recognized the binding sites in AKT2 3'UTR and directly repressed AKT2 expression through mRNA degradation and protein synthesis inhibition.

\section{AKT2 is up-regulated in gastric cancer and shows negative correlation with let-7b/g}

Expression of AKT2 mRNA was up-regulated in 8 gastric cancer cell lines except SNU1 compared with normal gastric mucosal sample (Figure 4A). In 28 pairs of primary gastric cancers, AKT2 mRNA expression showed upregulation in 19 (67.9\%) tumor samples by normalization with its expression in adjacent non-tumorous tissues. The expression of AKT2 in the cancer tissues showed higher expression than it expression in adjacent normal gastric tissues $(P=0.034$, Figure $4 \mathrm{~B})$. Strong AKT2 protein expression can be strongly detected in all 9 gastric cancer cell lines but only one normal gastric mucosal tissue showed week AKT2 protein expression (Figure 4C). In 28 paired primary protein samples, AKT2 protein expression showed up-regulated in the cancer tissues compared with adjacent non-tumorous tissues after quantification $(P=0.001$, Figure $4 \mathrm{D})$. We then examined the AKT2 mRNA and let-7b/g expression in 28 paired primary gastric cancer samples. The AKT2 mRNA expression was negatively correlated with let-7b/g expression (let-7b, $P=0.001$; let-7g, $P=0.021$; Figure $4 \mathrm{E}$ ), suggesting let-7b/g downregulation might be, at least partly, responsible for the aberrant activation of AKT2 in gastric cancers.

\section{siRNA-mediated knockdown of AKT2 phenocopies the tumor-suppressive effect of let-7b/g}

We have demonstrated that let-7b/g targeted AKT2 and exerted tumor suppressor function in gastric cancer. siRNAmediated AKT2 knockdown was therefore performed to investigate if AKT2 downregulation phenocopied the tumor-suppressive effect of let-7b/g. We transfected siAKT2 into gastric cancer cell lines AGS, NCI-N87 and MKN45. Successful AKT2 knockdown was confirmed by Western blot analysis. p-AKT (S473) and pS6 protein showed decreased expression upon siAKT2 transfection (Figure 5A). AKT2 knockdown suppressed AGS, NCI-N87 and MKN45 cell proliferation in a 5 -day MTT assays $(P<0.001$, Figure 5B). AKT2 knockdown also decreased monolayer colony formation in gastric cancer cells to a significant level (Figure 5C). Moreover, siAKT2 inhibited cell invasion of gastric cancer cells $(P<0.001$, Figure 5D). The effect of siAKT2 on tumor growth in vivo was also investigated.
The siAKT2-MKN45 and vector control cells were injected into nude mice subcutaneously. The tumor growth in siAKT2 groups was significantly decreased compared with the vector control after 22 days $(P<0.05$, Figure $5 \mathrm{E})$.

\section{AKT2 re-expression abrogated the growth-inhibitory effect of let-7b/g}

Since AKT2 is a predicted target of let- $7 \mathrm{~b} / \mathrm{g}$, we further investigated if AKT2 re-expression diminished the suppressive phenotype change caused by let-7b and let-7g. Interestingly, we found that the growth inhibitory effect of let-7b/g were partially abrogated by AKT2 re-expression (MTT proliferation assays, Figure 6A; monolayer colony formation assays, Figure 6B), suggesting AKT2 was functionally involved in let-7b/g-inducing suppression of gastric cancer cell growth. In vivo experiments yielded similar results. Cells co-transfected with let-7b/g and AKT2 formed bigger xenografts than those with let$7 \mathrm{~b} / \mathrm{g}$ alone $(P<0.001$, Figure $6 \mathrm{C})$.

\section{Discussion}

let-7 family, which has 10 mature subtypes identified (from 7a to 7i, miR-98 and miR-202), is closely associated with normal development and human cancers [24]. LIN28 and LIN28B have been found to act as post-transcriptional repressors of let-7 biogenesis by binding with the loop portion of the pri-let-7 hairpin and the stem of pre-let-7 to inhibit its binding with Drosha or Dicer [25]. Genomic DNA copy number alterations [26] and DNA methylation [27] are also responsible for the decreased expression of let-7 family in cancers. In 9 gastric cancer cells, we compared let-7b (in 22q13) and let-7g (in 3p21) expression with the DNA copy number change of their loci and found although the $P$-value is not significant due the limited number of cell lines, let-7b/g expression showed a trend of positive correlation with their array-CGH results (let-7b, $P=0.205$; let-7g, $P=0.088$; Additional file 3: Figure S1).

The let-7 miRNA family is involved in the proliferation, apoptosis and invasion of cancer cells. A number of studies have reported that let- 7 is down-regulated and acts as a tumor suppressor in kinds of cancer types, including nonsmall cell lung carcinoma [28], breast cancer [29], prostate cancer [30], nasopharyngeal carcinoma [31], hepatocellular carcinoma [32] and esophageal squamous cell carcinoma. Several oncogenes and signaling pathways, such as RAS oncogene, c-Myc [33], HMGA2 [15] and JAK-STAT3 pathway [24], are the main targets of let-7 in carcinogenesis. In addition, SNP rs61764370 in KRAS 3'UTR (T. $>$ G) promotes cell proliferation through downregulation of let-7a/b/c [34]. In current study, we not only confirmed the tumor suppressor function of let- $7 \mathrm{~b} / \mathrm{g}$ but also revealed a novel functional target of let-7 family, AKT2, together with the dysregulated signaling pathway, AKT2-mTOR-pS6. Ectopic expression of let-7b/g 


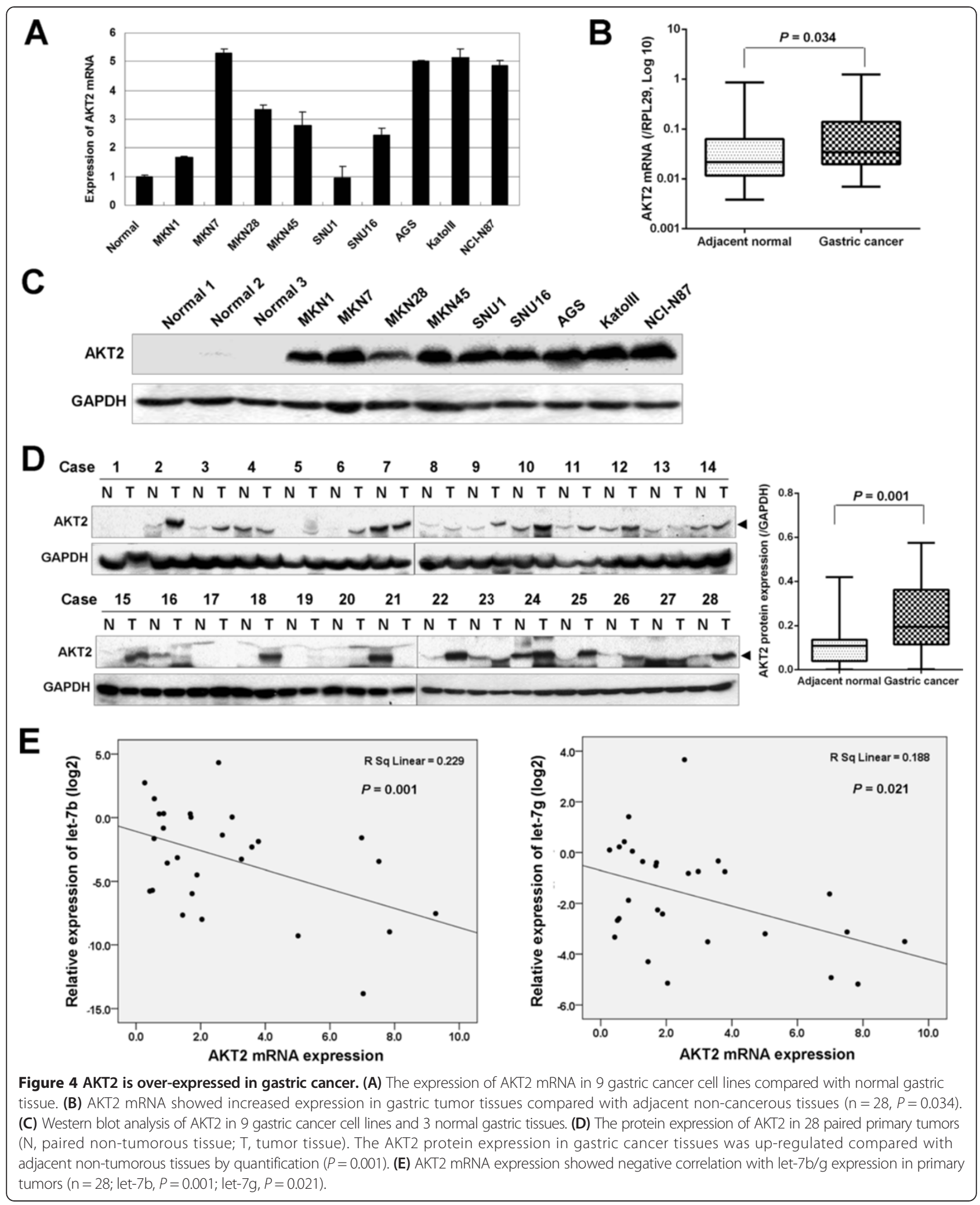

down-regulated AKT2 and its downstream effector pS6. In addition, AKT2 re-expression alleviated the suppressive phenotypes of let-7b/g, thus establishing their functional interaction. More importantly in primary samples, AKT2 mRNA expression showed a negative correlation with let-7b/g expression, suggesting that let-7b/g exerts its 

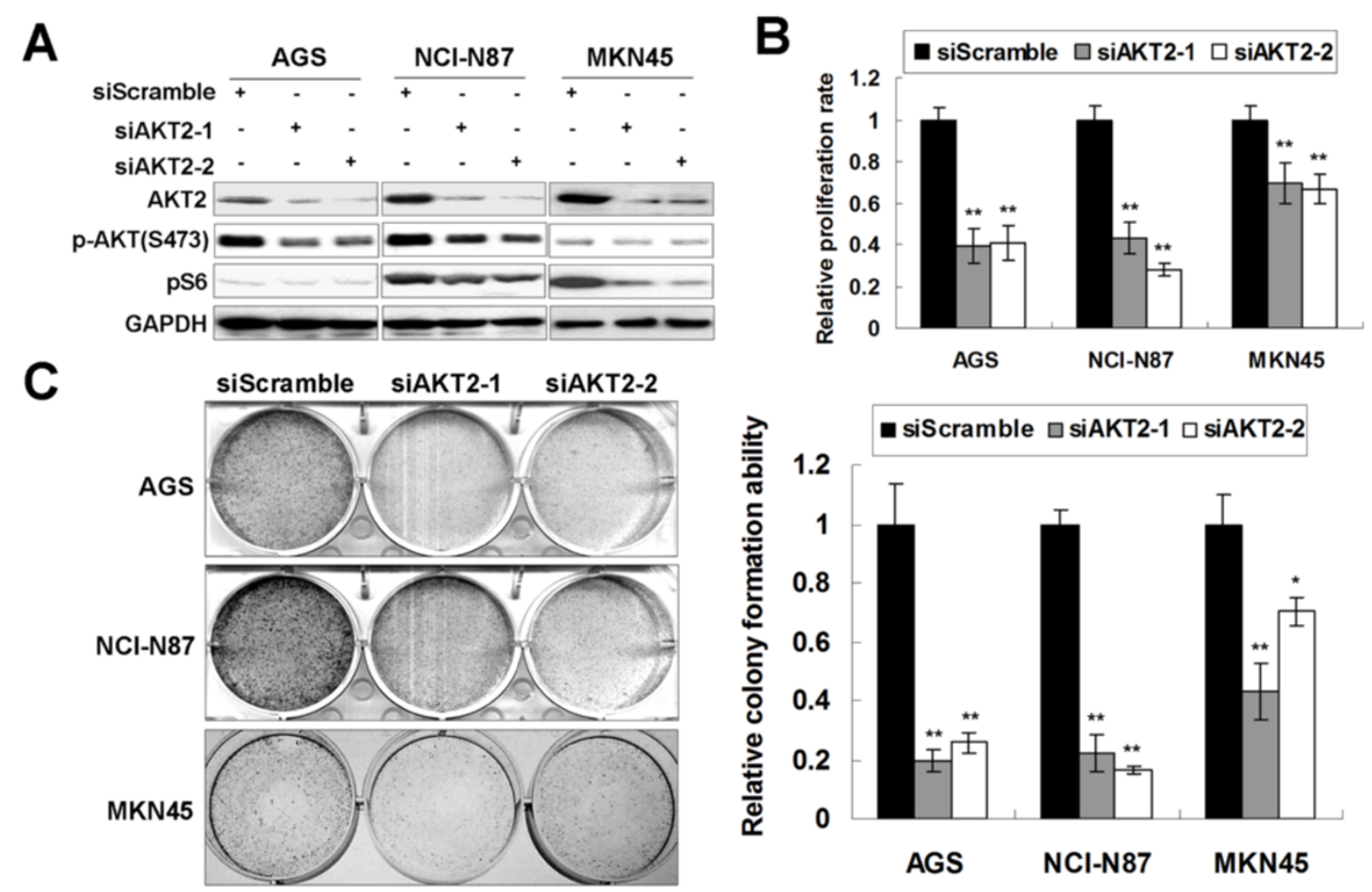

D
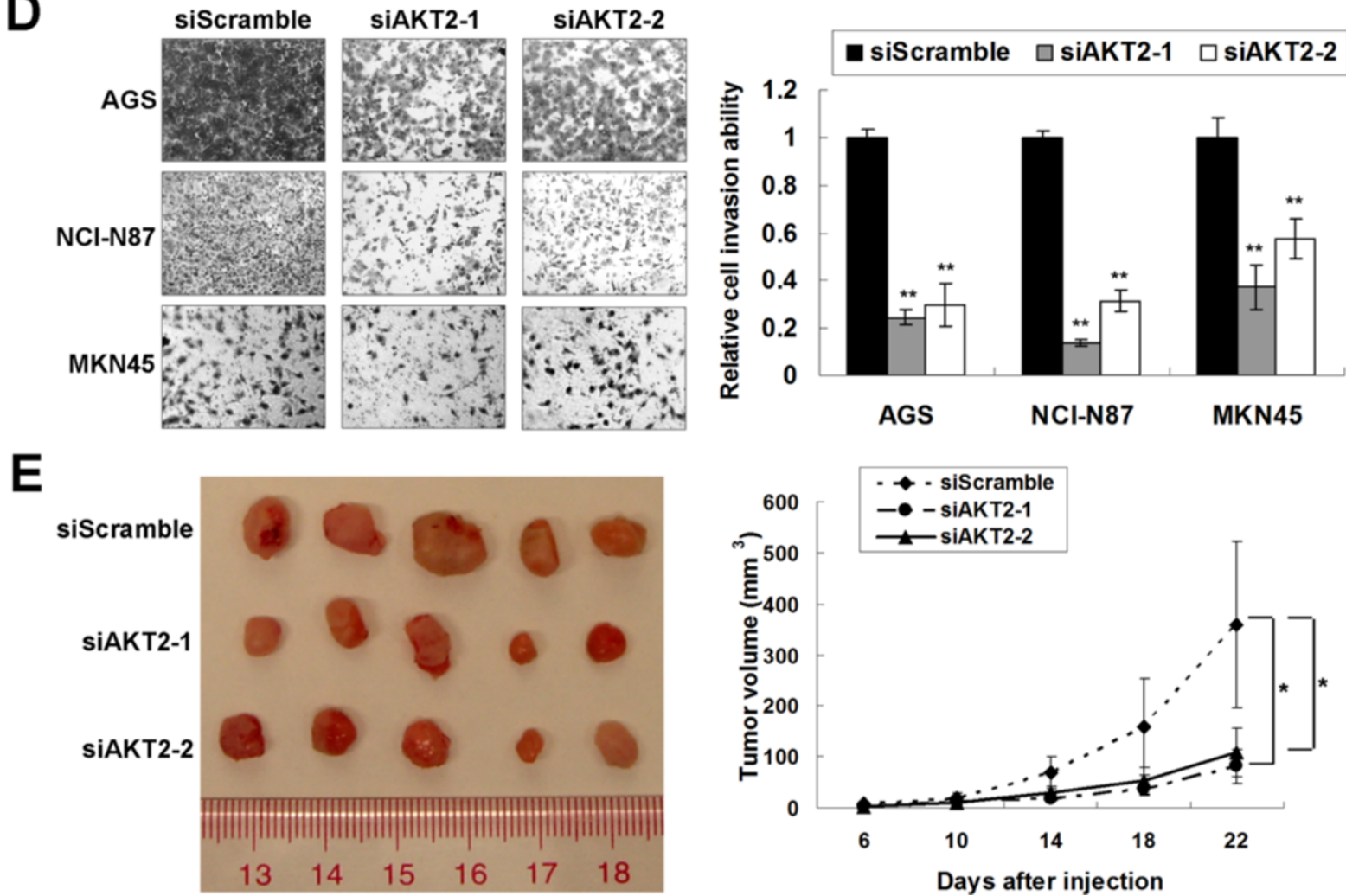

Figure $\mathbf{5}$ (See legend on next page.) 
(See figure on previous page.)

Figure 5 AKT2 knockdown phenocopies the tumor-suppressive effect of let-7b/g in gastric cancer cells. (A) Western blot of AKT2, p-AKT (S473) and pS6 after siAKT2 transfection in AGS, NCI-N87 and MKN45 cells. (B) AKT2 knockdown suppressed cell proliferation (**, P<0.001). 6 wells were measured for each group to get SDs. (C) AKT2 knockdown decreased monolayer colony formation in AGS, NCI-N87 and MKN45 cells (**, $P<0.001)$. The experiments was repeated in 3 wells to get SDs. (D) siAKT2 inhibited cell invasion of gastric cancer cells $\left({ }^{*}, P<0.001\right)$. The invaded cells in 3 random vision fields were counted for SDs achieving. (E) siAKT2 suppressed the formation of xenografts in nude mice compared with siScramble group $(*, P<0.05)$.

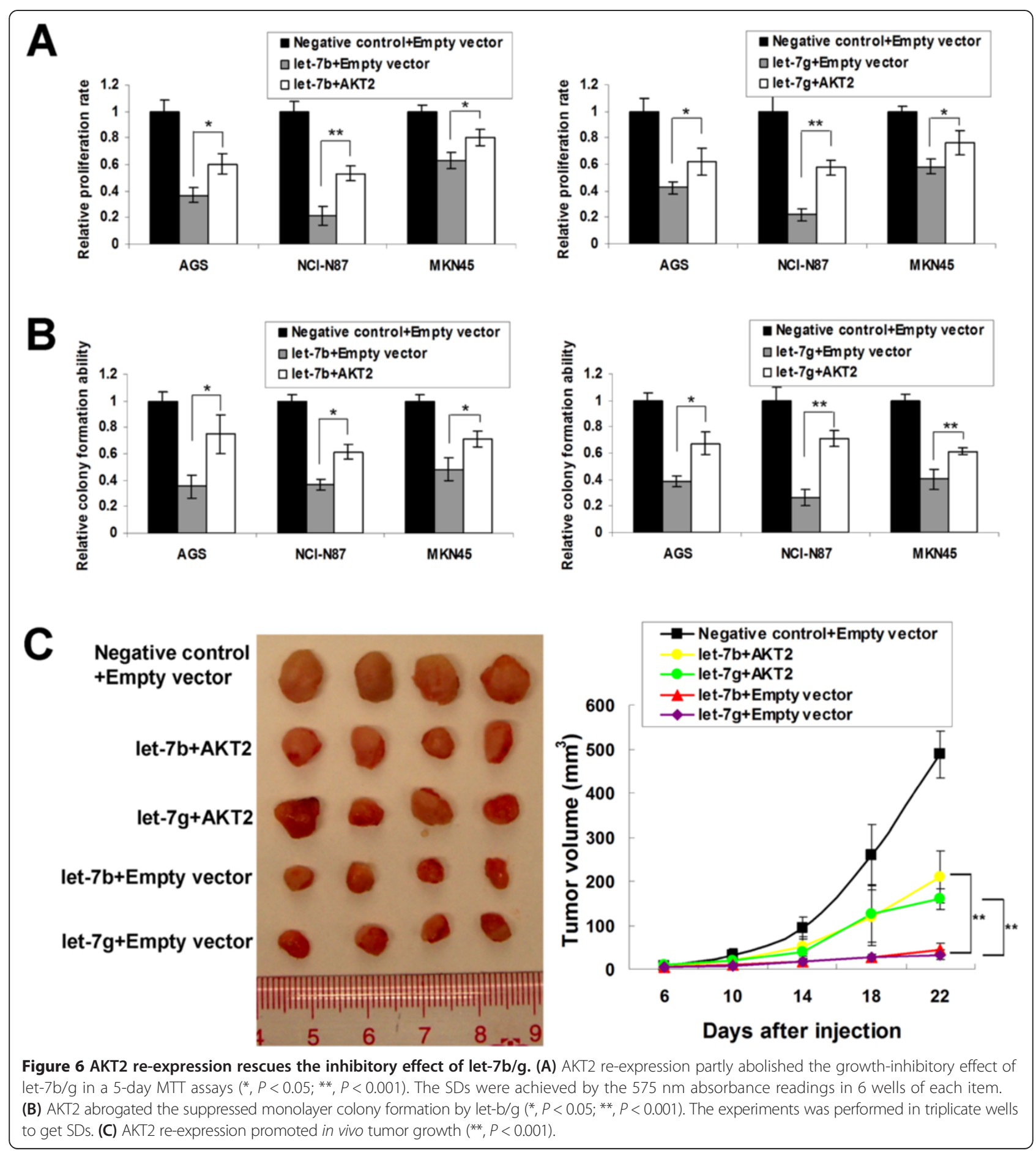


tumor suppressor function in gastric carcinogenesis at least by partly down-regulating AKT2. All these findings in this part revealed the critical role of let- $7 \mathrm{~b} / \mathrm{g}$ in molecular gastric carcinogenesis.

AKT2, a member of Protein Kinase B family, is an important signaling molecule in the insulin signaling pathway. It has been identified as an oncogene and amplified in human ovarian carcinomas [35], pancreatic carcinomas [36] and pancreatic ductal adenocarcinomas [37]. AKT2 is a link between the metabolic action of insulin signal transduction and tumorigenesis in liver malignancies [38]. As a major downstream effector of PI3K/AKT survival pathway, AKT2 expression markedly increased the incidence of pulmonary metastases in breast tumor model [39]. AKT2 knockdown by RNA interference suppresses cell proliferation and induces apoptosis even increases chemosensitivity in lung adenocarcinoma [40], malignant gliomas $[41,42]$, pancreatic cancer [43] and ovarian cancer [44]. We showed that AKT2 was upregulated in gastric cancer and its knockdown suppressed gastric cancer cell proliferation, reduced monolayer colony formation and inhibited xenograft formation in vivo, resembling the tumor-suppressive effects of let-7b/g in gastric cancer cells.

In conclusion, our results underscore let- $7 \mathrm{~b} / \mathrm{g}$ as important tumor-suppressive miRNAs in gastric cancer cells by directly targeting AKT2. All these findings support that the frequently down-regulated let-7b/g contributes to activation of AKT2 and gastric carcinogenesis which might has therapeutic potential in gastric cancer.

\section{Additional files}

Additional file 1: Table S1. Relative expression of let-7 family in gastric cancer cell lines compared with normal gastric tissue (from microRNA expression microarray data, $\log 2$ ratio).

Additional file 2: Table S2. Correlation of let-7b and let-7g expression with clinicopathologic features (significant $P$-value in bold and Italic format).

Additional file 3: Figure S1. The correlation of let-7b/g expression with DNA copy number change of its loci in gastric cancer cell lines $(n=9)$. (A) The correlation of let-7b expression with copy number change of $22 q 13$ ( $P=0.205$ ). (B) let-7g expression showed a trend of positive correlation with its copy number change in $3 p 21(P=0.088)$.

\section{Competing interests}

The authors declare that they have no competing interests.

\section{Authors' contributions}

WK, JHMT, RWML, YJD, WQY, YP carried out the experimental studies, interpreted the data, performed the statistical analysis. KML, JY, ASLC provided experimental materials. WK, JHMT and KFT contributed to the study design, manuscript drafting and provided fund for this study. All authors read and approved the final manuscript.

\section{Acknowledgements}

This work was supported by collaborative research fund from the Hong Kong Research Grants Council (CUHK8/CRF/11R) and National Natural Science Foundation of China (81201591).

\section{Author details}

${ }^{1}$ Department of Anatomical and Cellular Pathology, State Key Laboratory in Oncology in South China, Prince of Wales Hospital, The Chinese University of Hong Kong, Hong Kong, SAR, People's Republic of China. ${ }^{2}$ Institute of Digestive Disease, Partner State Key Laboratory of Digestive Disease, The Chinese University of Hong Kong, Hong Kong, SAR, People's Republic of China. ${ }^{3}$ Li Ka Shing Institute of Health Science, Sir Y.K. Pao Cancer Center, The Chinese University of Hong Kong, Hong Kong, SAR, People's Republic of China. ${ }^{4}$ Shenzhen Research Institute, The Chinese University of Hong Kong, Shenzhen, People's Republic of China. ${ }^{5}$ Department of Medicine and Therapeutics, The Chinese University of Hong Kong, Hong Kong, People's Republic of China. 'School of Biomedical Sciences, The Chinese University of Hong Kong, Hong Kong, People's Republic of China.

Received: 6 June 2014 Accepted: 24 September 2014

Published online: 05 October 2014

\section{References}

1. Parkin DM, Bray F, Ferlay J, Pisani P: Global cancer statistics, 2002. CA Cancer J Clin 2005, 55:74-108.

2. Hatakeyama M: Helicobacter pylori CagA - a bacterial intruder conspiring gastric carcinogenesis. Int J Cancer 2006, 119:1217-1223.

3. Lauren P: The Two Histological Main Types of Gastric Carcinoma: Diffuse and So-Called Intestinal-Type Carcinoma. An Attempt at a Histo-Clinical Classification. Acta Pathol Microbiol Scand 1965, 64:31-49.

4. Grabsch HI, Tan P: Gastric cancer pathology and underlying molecular mechanisms. Dig Surg 2013, 30:150-158.

5. Chen K, Rajewsky N: The evolution of gene regulation by transcription factors and microRNAs. Nat Rev Genet 2007, 8:93-103.

6. Bartel DP: MicroRNAs: target recognition and regulatory functions. Cell 2009, 136:215-233.

7. Lu J, Getz G, Miska EA, Alvarez-Saavedra E, Lamb J, Peck D, Sweet-Cordero A, Ebert BL, Mak RH, Ferrando AA, Downing JR, Jacks T, Horvitz HR, Golub TR: MicroRNA expression profiles classify human cancers. Nature 2005, 435:834-838.

8. Zhou C, Li X, Zhang X, Liu X, Tan Z, Yang C, Zhang J: microRNA-372 maintains oncogene characteristics by targeting TNFAIP1 and affects NFkappaB signaling in human gastric carcinoma cells. Int J Oncol 2013, 42:635-642.

9. Zhi Q, Guo X, Guo L, Zhang R, Jiang J, Ji J, Zhang J, Chen X, Cai Q, Li J, Liu B, Zhu Z, Yu Y: Oncogenic miR-544 is an important molecular target in gastric cancer. Anticancer Agents Med Chem 2013, 13:270-275.

10. Zhang X, Li X, Tan Z, Liu X, Yang C, Ding X, Hu X, Zhou J, Xiang S, Zhou C, Zhang J: MicroRNA-373 is upregulated and targets TNFAIP1 in human gastric cancer, contributing to tumorigenesis. Oncol Lett 2013, 6:1427-1434.

11. Zheng L, Qi T, Yang D, Qi M, Li D, Xiang X, Huang K, Tong Q: microRNA-9 suppresses the proliferation, invasion and metastasis of gastric cancer cells through targeting cyclin D1 and Ets1. PLoS One 2013, 8:e55719.

12. Zhao Y, Li C, Wang M, Su L, Qu Y, Li J, Yu B, Yan M, Yu Y, Liu B, Zhu Z: Decrease of miR-202-3p expression, a novel tumor suppressor, in gastric cancer. PLOS One 2013, 8:e69756.

13. Zhao X, Dou W, He L, Liang S, Tie J, Liu C, Li T, Lu Y, Mo P, Shi Y, Wu K, Nie $Y$, Fan D: MicroRNA-7 functions as an anti-metastatic microRNA in gastric cancer by targeting insulin-like growth factor-1 receptor. Oncogene 2013, 32:1363-1372.

14. Zhang L, Liu X, Jin H, Guo X, Xia L, Chen Z, Bai M, Liu J, Shang X, Wu K, Pan Y, Fan D: miR-206 inhibits gastric cancer proliferation in part by repressing cyclinD2. Cancer Lett 2013, 332:94-101.

15. Motoyama K, Inoue H, Nakamura Y, Uetake H, Sugihara K, Mori M: Clinical significance of high mobility group A2 in human gastric cancer and its relationship to let-7 microRNA family. Clin Cancer Res 2008, 14:2334-2340.

16. Matsushima $K$, Isomoto $H$, Inoue N, Nakayama T, Hayashi T, Nakayama M Nakao K, Hirayama T, Kohno S: MicroRNA signatures in Helicobacter pylori-infected gastric mucosa. Int J Cancer 2011, 128:361-370.

17. Hayashi Y, Tsujii M, Wang J, Kondo J, Akasaka T, Jin Y, Li W, Nakamura T, Nishida T, lijima H, Tsuji S, Kawano S, Hayashi N, Takehara T: CagA mediates epigenetic regulation to attenuate let-7 expression in Helicobacter pylori-related carcinogenesis. Gut 2013, 62:1536-1546.

18. Liang S, He L, Zhao X, Miao Y, Gu Y, Guo C, Xue Z, Dou W, Hu F, Wu K, Wu K, Nie $Y$, Fan D: MicroRNA let-7f inhibits tumor invasion and metastasis by targeting MYH9 in human gastric cancer. PLoS One 2011, 6:e18409. 
19. Kang W, Tong JH, Chan AW, Lung RW, Chau SL, Wong QW, Wong N, Yu J, Cheng AS, To KF: Stathmin1 plays oncogenic role and is a target of microRNA-223 in gastric cancer. PLoS One 2012, 7:e33919.

20. Rho HW, Lee BC, Choi ES, Choi IJ, Lee YS, Goh SH: Identification of valid reference genes for gene expression studies of human stomach cancer by reverse transcription-qPCR. BMC Cancer 2010, 10:240.

21. Kang W, Tong JH, Chan AW, Lee TL, Lung RW, Leung PP, So KK, Wu K, Fan D, Yu J, Sung JJ, To KF: Yes-associated protein 1 exhibits oncogenic property in gastric cancer and its nuclear accumulation associates with poor prognosis. Clin Cancer Res 2011, 17:2130-2139.

22. Lung RW, Tong JH, Sung YM, Leung PS, Ng DC, Chau SL, Chan AW, Ng EK, Lo KW, To KF: Modulation of LMP2A expression by a newly identified EpsteinBarr virus-encoded microRNA miR-BART22. Neoplasia 2009, 11:1174-1184.

23. Kang W, Tong JH, Chan AW, Zhao J, Dong Y, Wang S, Yang W, Sin FM, Ng SS, Yu J, Cheng AS, To KF: Yin Yang 1 contributes to gastric carcinogenesis and its nuclear expression correlates with shorter survival in patients with early stage gastric adenocarcinoma. J Transl Med 2014, 12:80.

24. Wang X, Cao L, Wang Y, Liu N, You Y: Regulation of let-7 and its target oncogenes (Review). Oncol Lett 2012, 3:955-960.

25. Piskounova E, Polytarchou C, Thornton JE, LaPierre RJ, Pothoulakis C, Hagan JP, lliopoulos D, Gregory Rl: Lin28A and Lin28B inhibit let-7 microRNA biogenesis by distinct mechanisms. Cell 2011, 147:1066-1079.

26. Wang Y, Hu X, Greshock J, Shen L, Yang X, Shao Z, Liang S, Tanyi IL, Sood AK, Zhang L: Genomic DNA copy-number alterations of the let-7 family in human cancers. PLoS One 2012, 7:e44399.

27. Lu L, Katsaros D, de la Longrais IA, Sochirca O, Yu H: Hypermethylation of let-7a-3 in epithelial ovarian cancer is associated with low insulin-like growth factor-II expression and favorable prognosis. Cancer Res 2007, 67:10117-10122.

28. Zhao B, Han H, Chen J, Zhang Z, Li S, Fang F, Zheng Q, Ma Y, Zhang J, Wu N, Yang $Y$ : MicroRNA let-7c inhibits migration and invasion of human non-small cell lung cancer by targeting ITGB3 and MAP4K3. Cancer Lett 2014, 342:43-51.

29. Ma L, Li GZ, Wu ZS, Meng G: Prognostic significance of let-7b expression in breast cancer and correlation to its target gene of BSG expression. Med Oncol 2014, 31:773.

30. Nadiminty N, Tummala R, Lou W, Zhu Y, Zhang J, Chen X, eVere White RW, Kung HJ, Evans CP, Gao AC: MicroRNA let-7c suppresses androgen receptor expression and activity via regulation of Myc expression in prostate cancer cells. J Biol Chem 2012, 287:1527-1537.

31. Wong TS, Man OY, Tsang CM, Tsao SW, Tsang RK, Chan JY, Ho WK, Wei WI, To VS: MicroRNA let-7 suppresses nasopharyngeal carcinoma cells proliferation through downregulating c-Myc expression. J Cancer Res Clin Oncol 2011, 137:415-422.

32. Zhu XM, Wu LJ, Xu J, Yang R, Wu FS: Let-7c microRNA expression and clinical significance in hepatocellular carcinoma. J Int Med Res 2011, 39:2323-2329.

33. Lan FF, Wang H, Chen YC, Chan CY, Ng SS, Li K, Xie D, He ML, Lin MC, Kung HF: Hsa-let-7g inhibits proliferation of hepatocellular carcinoma cells by downregulation of c-Myc and upregulation of p16(INK4A). Int J Cancer 2011, 128:319-331.

34. Crowley EH, Arena S, Lamba S, Di Nicolantonio F, Bardelli A: Targeted knock-in of the polymorphism rs61764370 does not affect KRAS expression but reduces let-7 levels. Hum Mutat 2014, 35:208-214.

35. Cheng JQ, Godwin AK, Bellacosa A, Taguchi T, Franke TF, Hamilton TC, Tsichlis PN, Testa JR: AKT2, a putative oncogene encoding a member of a subfamily of protein-serine/threonine kinases, is amplified in human ovarian carcinomas. Proc Natl Acad Sci U S A 1992, 89:9267-9271.

36. Cheng JQ, Ruggeri B, Klein WM, Sonoda G, Altomare DA, Watson DK, Testa JR: Amplification of AKT2 in human pancreatic cells and inhibition of AKT2 expression and tumorigenicity by antisense RNA. Proc Natl Acad Sci U S A 1996, 93:3636-3641.

37. Ruggeri BA, Huang L, Wood M, Cheng JQ, Testa JR: Amplification and overexpression of the AKT2 oncogene in a subset of human pancreatic ductal adenocarcinomas. Mol Carcinog 1998, 21:81-86.

38. Nemazanyy I, Espeillac C, Pende M, Panasyuk G: Role of PI3K, mTOR and Akt2 signalling in hepatic tumorigenesis via the control of PKM2 expression. Biochem Soc Trans 2013, 41:917-922.

39. Dillon RL, Marcotte R, Hennessy BT, Woodgett JR, Mills GB, Muller WJ: Akt1 and akt2 play distinct roles in the initiation and metastatic phases of mammary tumor progression. Cancer Res 2009, 69:5057-5064.
40. Sithanandam G, Fornwald LW, Fields JR, Morris NL, Anderson LM: Anti-tumor efficacy of naked siRNAs for ERBB3 or AKT2 against lung adenocarcinoma cell xenografts. Int J Cancer 2012, 130:251-258.

41. Cui Y, Wang Q, Wang J, Dong Y, Luo C, Hu G, Lu Y: Knockdown of AKT2 expression by RNA interference inhibits proliferation, enhances apoptosis, and increases chemosensitivity to the anticancer drug VM-26 in U87 glioma cells. Brain Res 2012, 1469:1-9.

42. Zhang B, Gu F, She C, Guo H, Li W, Niu R, Fu L, Zhang N, Ma Y: Reduction of Akt2 inhibits migration and invasion of glioma cells. Int J Cancer 2009, 125:585-595.

43. Shi XH, Liang ZY, Ren XY, Liu TH: Combined silencing of K-ras and Akt2 oncogenes achieves synergistic effects in inhibiting pancreatic cancer cell growth in vitro and in vivo. Cancer Gene Ther 2009, 16:227-236.

44. Noske A, Kaszubiak A, Weichert W, Sers C, Niesporek S, Koch I, Schaefer B, Sehouli J, Dietel M, Lage H, Denkert C: Specific inhibition of AKT2 by RNA interference results in reduction of ovarian cancer cell proliferation: increased expression of AKT in advanced ovarian cancer. Cancer Lett 2007, 246:190-200.

doi:10.1186/s12967-014-0281-3

Cite this article as: Kang et al:: let-7b/g silencing activates AKT signaling to promote gastric carcinogenesis. Journal of Translational Medicine $201412: 281$

\section{Submit your next manuscript to BioMed Central and take full advantage of:}

- Convenient online submission

- Thorough peer review

- No space constraints or color figure charges

- Immediate publication on acceptance

- Inclusion in PubMed, CAS, Scopus and Google Scholar

- Research which is freely available for redistribution 